\title{
Quantum Interference and Inelastic Scattering in a Model Which-Way Device
}

\author{
Angus MacKinnon ${ }^{1,2, *}$ and Andrew D. Armour ${ }^{3}$ \\ 1 The Blackett Laboratory, Imperial College London, London SW7 2BW, UK \\ 2 The Cavendish Laboratory, Madingley Rd, Cambridge CB3 OHE, UK \\ 3 School of Physics and Astronomy, University of Nottingham, NG7 2RD, UK
}

(Received November 21, 2018)

\begin{abstract}
A which-way device is one which is designed to detect which of 2 paths is taken by a quantum particle, whether Schrödinger's cat is dead or alive. One possible such device is represented by an Aharonov-Bohm ring with a quantum dot on one branch. A charged cantilever or spring is brought close to the dot as a detector of the presence of an electron. The conventional view of such a device is that any change in the state of the cantilever implies a change in the electron state which will in turn destroy the interference effects. In this paper we show that it is in fact possible to change the state of the oscillator while preserving the quantum interference phenomenon.
\end{abstract}

KEYWORDS: NEMS; mesoscopics; Aharonov-Bohm; which-way device

\section{Introduction}

The concept of a "which-way" device has always played an important role in our understanding of quantum mechanics, representing as it does one of the most difficult concepts in modern physics: Schrödinger's famous cat is neither alive nor dead. Textbooks of quantum mechanics typically discuss electrons going through a double slit and state that any attempt to identify which slit the electron goes through will result in the destruction of the interference pattern associated with the double slit. ${ }^{1)}$

In a recent experiment, ${ }^{2)}$ Buks et al. used a "whichway" device to probe dephasing effects in mesoscopic electronic systems. They fabricated an Aharonov-Bohm ring $^{3,4)}$ in a semiconductor structure with a quantum dot included in one of the arms. A quantum point contact (QPC) was fabricated adjacent to the dot so that the current flowing through it was modulated when the nearby dot was occupied. In this system the current through the QPC effectively measures the path taken by the electron (by measuring the occupancy of the adjacent quantum dot). Measurement of the path taken by an electron in an Aharonov-Bohm ring inevitably leads to dephasing of the electrons, and hence suppression of the interference fringes in the current as a function of flux.

Recently, Armour and Blencowe ${ }^{5,6)}$ discussed the electromechanical "which-path" device illustrated in figure 1. This device is based on the system investigated by Buks et al., but with the QPC replaced by a micron-sized cantilever, positioned close to the dot. The cantilever can be coated in a thin metal layer so that it carries a net charge and therefore interacts with charges on the dot. ${ }^{7)}$ The presence of an additional electron on the dot couples effectively to just the fundamental flexural mode of the charged cantilever, ${ }^{5}$ ) causing a deflection. For low enough temperatures, and for high enough resonant frequencies, the fundamental mode of the cantilever can be treated as a quantum harmonic oscillator and coherent

* Present address: Blackett Lab., Imperial College London, London SW7 2BW, UK; E-mail: a.mackinnon@imperial.ac.uk

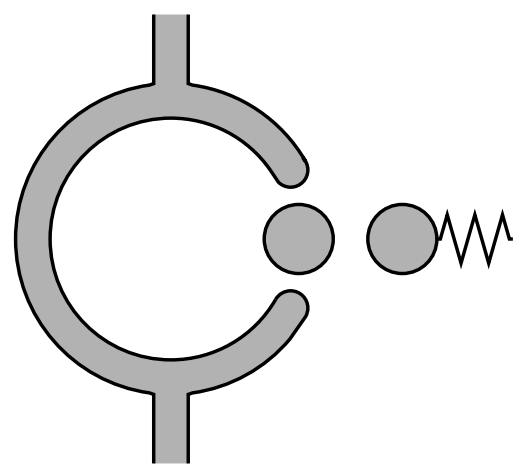

Fig. 1. An Aharonov-Bohm ring containing a quantum dot in close proximity to another charged dot attached to a spring or cantilever.

coupling between the electrons and the cantilever must be considered. ${ }^{5)}$

Considering a cantilever coupled to the dot rather than a QPC is of interest for two main reasons. Firstly, on a practical level, coupling to an Aharonov-Bohm ring may provide a very effective way of probing quantum behaviour in micro-mechanical systems. ${ }^{8)}$ Secondly, because the problem reduces to that of coupling to a quantum harmonic oscillator, it provides a generic model for investigating systems in which the electrons couple to a single, localised degree of freedom (in contrast to the many, transient, degrees of freedom associated with electrons passing through a QPC).

The conventional description of what happens in the electromechanical "which-path" device would be that the electrons are dephased whenever their interaction with the cantilever results in a change in the state of the cantilever. However, this presupposes that the electrons travel no more than half-way around the ring before leaving at the top junction so that only one of the two interfering paths passes through the dot. In practice, for a device in which the only connections between the ring and the outside world occur via the top and bottom leads, ${ }^{9}$ ) many electron paths contribute to the current through the device, some of which travel around 
the ring several times (therefore passing through the dot several times). ${ }^{10)}$

The presence of interfering paths which each pass through the quantum dot leads to interesting effects which arise from the fact that the electrons can couple to the cantilever coherently. Because the electrons on the dot interact effectively with just the fundamental flexural mode of the cantilever, ${ }^{5)}$ it is possible for electrons passing through the dot on different paths to change the state of the cantilever in the same way and so remain phase coherent.

The idea that electrons can undergo inelastic scattering and still remain phase coherent goes against the intuitive picture developed in many text-books that a change of state in the measuring device or environment always causes dephasing. However, it has been recognised for some time ${ }^{11)}$ that exchange of energy is neither a necessary nor a sufficient condition for dephasing.

In this paper we develop a numerical simulation to investigate the nature of the interference fringes in the current as a function of the magnetic flux when the electron paths can thread the ring more than once. In particular we explore the contribution to the fringes arising from interfering electron paths which include inelastic scattering.

\section{The Model}

The system may be described by a Hamiltonian of the form

$$
\begin{aligned}
H= & -E_{\mathrm{e}}\left(\frac{\partial}{\partial x_{\mathrm{e}}}-e \frac{\Phi}{2 \pi R}\right)^{2} \\
& -E_{\mathrm{c}}\left(\frac{\partial^{2}}{\partial y_{\mathrm{c}}^{2}}-\frac{1}{4} y_{\mathrm{c}}^{2}\right)+V\left(x_{\mathrm{e}}, y_{\mathrm{c}}\right)
\end{aligned}
$$

where $x_{\mathrm{e}}$ and $y_{\mathrm{c}}$ are the electron and phonon (i.e. the fundamental flexural mode of the cantilever) coordinates respectively, $\Phi$ and $R$ are the magnetic flux and the radius of the ring and

$$
V\left(x_{\mathrm{e}}, y_{\mathrm{c}}\right)=\left\{\begin{array}{cc}
\alpha y_{\mathrm{c}} & \left|x_{\mathrm{e}}\right|<a \\
V_{\mathrm{b}} & a<\left|x_{\mathrm{e}}\right|<a+d, \\
0 & a+d<\left|x_{\mathrm{e}}\right|
\end{array},\right.
$$

which contains a pair of barriers of height $V_{\mathrm{b}}$ and width $d$ separated by $2 a$. The depth of the well is $\alpha y_{\mathrm{c}}$ which represents the coupling to the cantilever. Thus the effective potential inside the well may be written as

$$
V_{\mathrm{eff}}=\frac{E_{\mathrm{c}}}{4}\left[\left(y_{c}+\frac{2 \alpha}{E_{\mathrm{c}}}\right)^{2}-\left(\frac{2 \alpha}{E_{\mathrm{c}}}\right)^{2}\right]
$$

which is a harmonic potential shifted with respect to that outside the well. The matching of the wave functions at the boundaries of the well is most easily carried out by Fourier transforming with respect to $y_{\mathrm{c}}$ so that the $n^{-}$ phonon state inside of the well $\phi_{n}^{\text {well }}\left(k_{\mathrm{c}}\right)$ is related to that outside by

$$
\phi_{n}^{\text {well }}=\exp \left(\mathrm{i} k_{\mathrm{c}} \frac{2 \alpha}{E_{\mathrm{c}}}\right) \phi_{n}^{\text {out }}
$$

where the exponential can be turned into an operator in the phonon number representation by first noting that we can write

$$
\hat{k}_{c}=\frac{1}{2} \mathrm{i}\left(\hat{c}^{\dagger}-\hat{c}\right)
$$

where $\hat{c}^{\dagger}, \hat{c}$ are the phonon creation and annihilation operators. This operator may be written in matrix form and diagonalised, the exponential carried out on the eigenvalues, and the result transformed back into the phonon number representation. It is only necessary to perform this operation once, at the start of the calculation.

Writing the wave functions in the form

$$
\psi\left(x_{\mathrm{e}}, k_{\mathrm{c}}\right)=\sum_{n} \phi_{n}\left(k_{\mathrm{c}}\right)\left(a_{n}^{+} e^{\mathrm{i} k_{n}^{+} x_{\mathrm{e}}}+a_{n}^{-} e^{\mathrm{i} k_{n}^{-} x_{\mathrm{e}}}\right)
$$

where $k_{n}^{ \pm}$are the left and right-going wave vectors of the electronic part of the wave function when the cantilever is in the $n$-phonon state (in the absence of the magnetic flux $k_{n}^{-}=-k_{n}^{+}$) it is straightforward to derive expressions for the transmission and reflection matrices at various boundaries in the potential and to combine these into transmission and reflection coefficients, and hence scattering $(S)$ matrices describing the whole system.

At the junctions with the leads amplitude and current conservation are ensured by appropriate boundary conditions

$$
\begin{aligned}
& \psi_{1}=\psi_{2}=\psi_{3} \\
& \left.\frac{d \psi}{d x}\right|_{1}+\left.\frac{d \psi}{d x}\right|_{2}+\left.\frac{d \psi}{d x}\right|_{3}=0
\end{aligned}
$$

where the derivatives are all defined towards the junction. In terms of scattering matrices $\mathbf{S}$ which describe the coupling of incident and emitted waves we can combine the 2 branches into an Aharonov-Bohm loop using

$$
\Sigma_{12}=\Sigma_{1}+\Sigma_{2}
$$

where

$$
\boldsymbol{\Sigma}=(\mathbf{S}-1) *(\mathbf{S}+1)^{-1}
$$

\section{Results}

For orientation fig. 2 shows the transmission of the dot without the ring as a function of total energy for the parameters used throughout this paper. In particular the cantilever is initially in the 2 phonon state $(n=2)$ but the different curves correspond to different final states of the cantilever. Note the well defined resonant tunnelling peaks separated by the phonon energy $E_{\mathrm{ph}}$ corresponding to the bound state of the dot with $n$ phonons. Note also the strong mixing of the cantilever states illustrated by the presence of finite weight in all peaks for various final states. We have, of course, chosen a relatively strong coupling for the purpose of illustration, but not so strong as to introduce new physics.

Fig. 3 shows the transmission of the ring as a function of magnetic flux (in units of $h / e$ ) again with the cantilever initially in the 2 phonon state. The energy is 


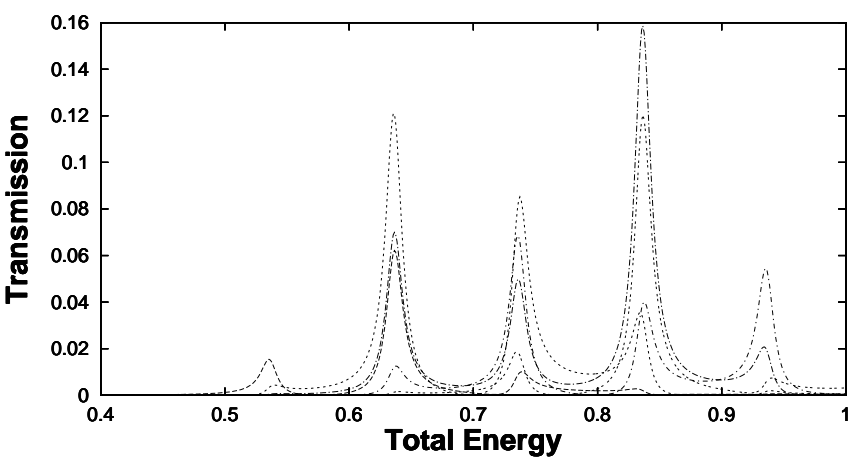

Fig. 2. Transmission coefficients of the dot as a function of total energy (electron + phonon) with the cantilever in initial state $n=2$. The various lines represent the final state of the cantilever $(n=0--, n=1 \quad--, n=2 \quad-\cdot-\cdot, n=3 \quad-\cdot, n=$ $4 \quad \cdots \quad \cdots)$. The parameters used are: $E_{\mathrm{e}}=1, E_{\mathrm{ph}}=0.1$, $\alpha=0.05, V_{b}=4, d=1,2 a=\pi$.

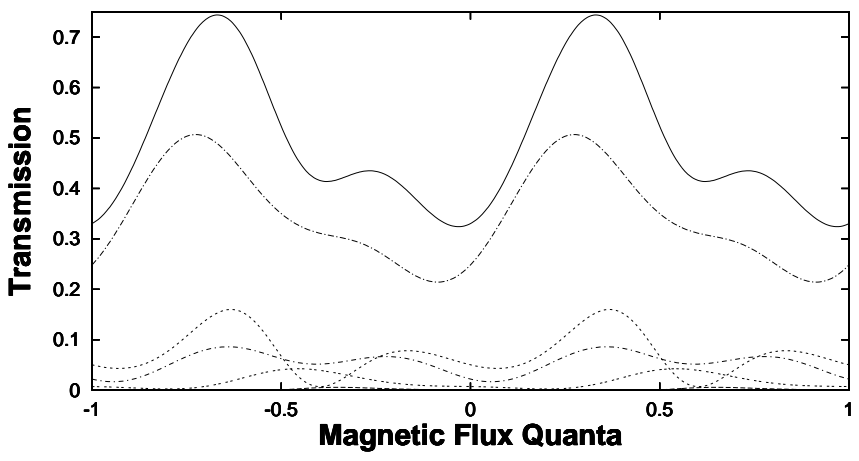

Fig. 3. Transmission coefficients of the the ring as a function of magnetic flux with the cantilever in an initial state with 2 phonons. The energy is 0.7365 , the maximum of the $3 \mathrm{rd}$ peak in fig. 2. ${ }^{12)}$ The labelling and parameters are as in fig. 2.

chosen as 0.7365 which is the maximum of the $3 \mathrm{rd}$ peak in fig. 2 corresponding to the bound state of the dot plus 2 phonons. Note that the $n=2$ line, which corresponds to the elastic transmission in which the state of the cantilever is unchanged, has higher values than the other curves but is still modulated by the magnetic field. In the absence of the cantilever and the ring the transmission here would be perfect. It is reduced from this value due to scattering by the cantilever and interference effects on the ring. This curve clearly contains components with period of 1 corresponding to the usual AharonovBohm effect ${ }^{3)}$ and usually interpreted as interference between the 2 different paths joining the source and the drain leads of the ring. The curve is far from perfectly sinusoidal however, indicating the presence of higher order contributions such as the weak localisation or correlated back scattering effect ${ }^{13)}$ corresponding to interference between paths round the whole ring in clockwise and anticlockwise directions respectively.

The curves in fig. 3 with different final states, corresponding to the creation or annihilation of one or more phonons have significant weight and, perhaps unexpectedly, are also modulated by the magnetic field. These results correspond to a situation in which the state of the cantilever has been changed but the transmission still shows interference effects. Is this consistent with the idea that a change of state of a measuring device causes dephasing and hence destroys interference? The naïve interpretation would be that the cantilever's change of state indicates that the electron must have gone through the dot and emerged with a different energy. This would not then interfere with a wave which has gone by the alternative path and has not gone through the dot.

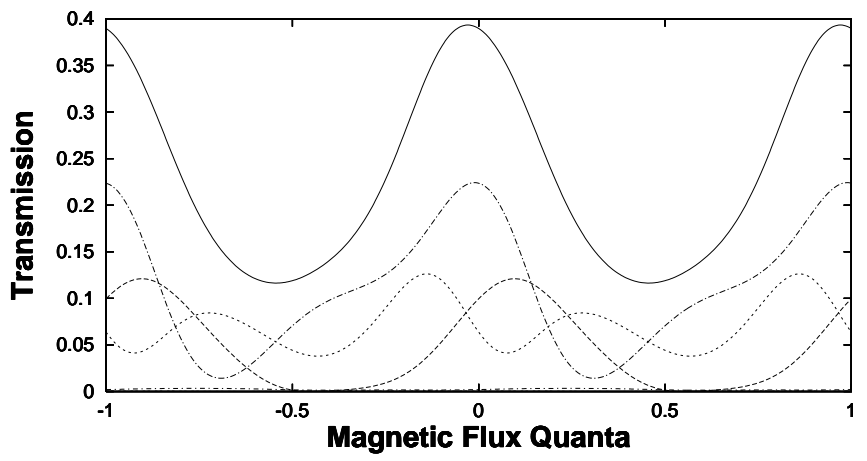

Fig. 4. Transmission coefficients of the the ring as a function of magnetic flux (in units of $h / e$ ) with the cantilever in an initial state with 2 phonons. The energy is 0.6365 , the maximum of the 2nd peak in fig. 2. ${ }^{12)}$ The labelling and parameters are as in fig. 2.

In order to investigate this further we change the energy to correspond to the 2 nd peak in fig. 2 at the bound state of the dot but with a single phonon. The initial state still has 2 phonons. Now we observe that there are several curves with similar average transmission probabilities and similar amplitudes of modulation. In fact no particular state is dominant. This is not so surprising given that the energy chosen corresponds to a different state of the dot than would correspond to the initial state of the cantilever. Both the $n=0$ and $n=2$ curves are dominated by contributions with periods of 1 flux quantum $h / e$, but the $n=1$ curve clearly has a significant contribution with $\frac{1}{2} h / e$.

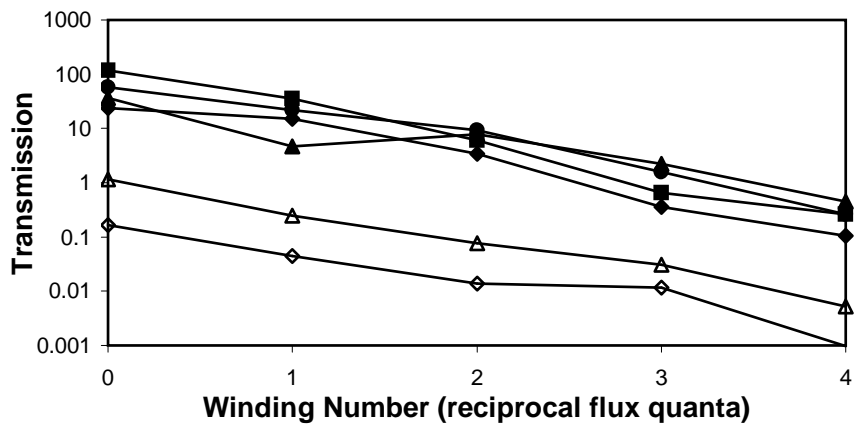

Fig. 5. The Fourier transform of fig. 4 plotted on a logarithmic scale for clarity. Note the presence of structure at various periods (units of $e / h$ ). Initial state $n=2$; total transmission squares, final state $n=0$ - filled diamond, $n=1$ - filled triangle, $n=2$ - filled circle, $n=3$ - open triangle, $n=4$ - open diamond. Note that the lines are a guide to the eye and should not be interpreted as intermediate values. As the calculation is performed with perfectly $1 \mathrm{D}$ wires the results are perfectly periodic and the Fourier transform is exactly zero between the plotted values.

This becomes clearer when the data in fig. 4 are Fourier transformed. In fig. 5 the $n=1$ results (filled dia- 
monds) have larger higher order components, but there are also significant contributions at other phonon numbers. Again we observe quantum interference in spite of the change of state of the cantilever.

\section{Analysis}
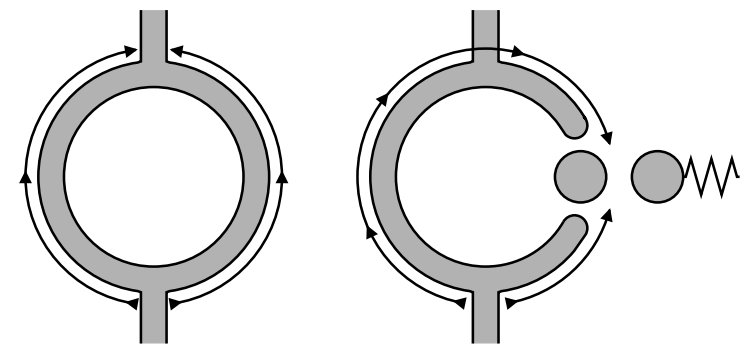

Fig. 6. Left: a conventional Aharonov-Bohm ring, with an indication of the 2 partial waves which interfere to give a conductance oscillation with period $h / e$. Right: the device discussed here, with an indication of the 2 partial waves which interfere at the quantum dot.

How is it possible for us to observe interference at the same time as a change of state of the cantilever? The first important observation is that the period of the most significant contribution is a single flux quantum $h / e$, similar to the original Aharonov-Bohm effect. ${ }^{3)}$ The "winding number" of the responsible interference process must therefore be one. The simplest such process involves interference between partial waves going clockwise and anti-clockwise at the dot, rather than at the source or drain of the ring (see figure 6 . Thus the amplitude of the wave function at the dot varies with a period of 1 flux quantum. The value of the matrix element coupling the electron to the cantilever is proportional to this amplitude such that the strength of the coupling between the electron and the cantilever also varies with period $h / e$. As this accounts for the basic periodicity of the process the waves emitted from the dot are not subject to any further interference and presumably may be described by a single wave travelling from the dot to the drain of the ring.

On the other hand a process might be considered by which a single wave travels from the source to the dot where a phonon is created or annihilated. The emitted wave may then split in 2 and travel either clockwise or anti-clockwise to the source or to the drain, where the 2 partial waves again interfere. Note that this process also has a winding number of unity.

A simple higher order process involves a combination of the above 2 paths; 2 paths from the source to the dot and 2 paths from the dot to the source or drain. In such a process the first step would have periodicity $h / e$ but the source of the 2nd step is itself modulated with the same periodicity. If the basic process is controlled by a term $\propto \cos \left(2 \pi \frac{e}{h} \Phi\right)$ then the 2 nd order one has an amplitude $\propto \cos ^{2}\left(2 \pi \frac{e}{h} \Phi\right)$ which has period $h / 2 e$.

It would be interesting to be able to filter out the elastic contribution. For the particular configuration discussed here a simple method for doing so would be to add an extra barrier to the drain lead with a height be- tween the $n=0$ and $n=1$ bound state energies of the dot. This would block the electron if it retains its initial energy but allow it through if it has gained $E_{\mathrm{c}}$ by annihilation of a phonon. More generally the behaviour of the system as a function of the height of such a barrier would be a useful probe of the effect.

\section{Discussion}

Why do these inelastic processes not destroy the quantum interference? In most discussions of dissipation the energy transfer is with a continuum such that the final state of the electron is also a continuum. ${ }^{14)}$ In the present case the coupling is to a single vibrational mode and the final state is therefore a linear combination of well defined separate states. Thus interference is still possible even after the energy has been changed. Of course the processes which destroy the conventional Aharonov-Bohm effects will still be operative here ${ }^{15)}$ and will tend to suppress higher order processes involving multiple circuits of the ring.

The fact that the transmission can be dominated by contributions involving a change in phonon number with a periodicity of one flux quantum means that the distance the electrons have to travel through the ring coherently is the same as for the usual elastic processes. This implies that the lowest-order inelastic interference effects should be no more difficult to observe than the elastic ones, discussed in detail by Armour and Blencowe. ${ }^{5)}$ The key practical difficulty is in obtaining reasonably strong coupling between the quantum dot and a cantilever of sufficiently high frequency that the thermal broadening of the electron energies in the leads does not exceed the energy of the phonons associated with the fundamental flexural mode.

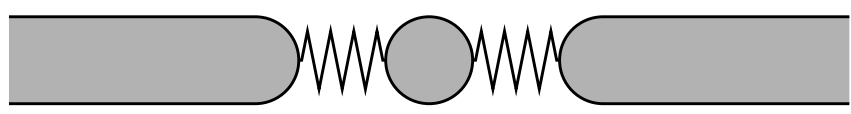

Fig. 7. An example of a quantum shuttle in which a quantum dot can oscillate between 2 contacts.

An interesting modification of the system we have considered here (shown in fig. 1) would be to combine the quantum oscillator and the dot into one, forming a quantum shuttle ${ }^{16)}$ as in fig. 7. Although the essential geometry of the system would remain the same, this alternative set-up might well make it easier to couple the electronic degrees of freedom to a very high frequency oscillator. ${ }^{17}$ )

We have shown that the which-way device involving coupling to a nanoscopic cantilever, which is a simple modification of an Aharonov-Bohm ring, has unexpected properties. It is possible to change the state of the detector, the cantilever, while retaining the quantum interference. This appears to contradict the statement contained in many textbooks that any attempt to determine which path the electron has taken will result in the destruction of the interference. However, the nature of the process involved is such that it actually tells us absolutely nothing about which path the electron has taken. In fact it confirms that the electron has taken both paths. Hence 
the conventional view is not wrong; it just requires a more subtle interpretation.

\section{Acknowledgements}

We would like to thank the EPSRC for financial support and the Cavendish Laboratory, Cambridge for its hospitality.

1) R. Feynman, R. Leighton and M. Sands: The Feynman Lectures on Physics (Addison-Wesley, MA, 1965), Vol. III.

2) E. Buks, R. Schuster, M. Heiblum, D. Mahalu and V. Umansky: Nature 391 (1998) 871.

3) Y. Aharonov and D. Bohm: Phys. Rev. 115 (1959) 485.

4) R. Webb, S. Washburn, C. Umbach and R. Laibowitz: Phys. Rev. Lett. 54 (1985) 2696.

5) A.D. Armour and M.P. Blencowe: Phys. Rev. B 64 (2001) 035311.

6) A.D. Armour and M.P. Blencowe: Physica B 316-317 (2002) 400

7) J. Kirschbaum, E.M. Höhberger, R.H. Blick, W. Wegscheider and M. Bichler: Appl. Phys. Lett. 81 (2002) 280

8) A.D. Armour, M.P. Blencowe and K.C. Schwab: Phys. Rev. Lett. 88 (2002) 148301.
9) Note that this is not the case in the experiment of Buks et al. and the device analysed by Armour and Blencowe.

10) A. Yacoby, R. Schuster and M. Heiblum: Phys. Rev. B 53 (1996) 9583.

11) Y. Imry: Introduction to Mesoscopic Physics (Oxford University Press, Oxford, U.K.) (1997) chap. 3.

12) Note that the quantity plotted here is $\left|t_{i j}\right|^{2}$ for electrons incident from one lead. This is equal to the corresponding quantity for electrons incident from the other lead only when the magnetic field is reversed. The conductivity contains the sum of both quantities and is thus symmetric in the field (Onsager relation).

13) A. MacKinnon: in Low-Dimensional Semiconductor Structures, eds. K. Barnham, D.D. Vvedensky (Cambridge University Press, Cambridge, U.K.) (2001) chap. 5.

14) A.O. Caldera and A.J. Leggett: Physica (Amsterdam) 121A (1983) 587.

15) A.E. Hansen, A. Kristensen, S. Pedersen, C.B. Sørensen and P.E. Lindelof: Phys. Rev. B 64 (2001) 045327.

16) A.D. Armour and A. MacKinnon: Phys. Rev. B 66 (2002) 035333.

17) A single-electron transistor in which electron motion through an individual $\mathrm{C}_{60}$ moleclue was coupled to a quantised vibrational degree of freedom with a frequency $\sim 1 \mathrm{THz}$ was demonstrated recently by H. Park, J. Park, A.K. Lim, E.H. Anderson, A.P. Alivisotos and P.L. McEuen: Nature 407 (2000) 57 